Inra Prod. Anim., 2009, 22 (4), 345-354

\section{Pratiques d'élevage dans les exploitations bovines allaitantes conduites sur pâturage dans les Hauts de la Réunion}

\author{
J.-P. CHOISIS 1,2 , P. GRIMAUD 2,3 , C. LASSALLE ${ }^{2}$ \\ ${ }^{1}$ INRA, UMR1201 Dynafor, F-31326 Castanet-Tolosan, France \\ 2 CIRAD, Pôle Elevage, 7 chemin IRAT, F-97410 Saint Pierre, France \\ ${ }^{3}$ CIRAD, Département Environnement et Sociétés, F-34032 Montpellier, France \\ Courriel : jean-philippe.choisis@toulouse.inra.fr
}

A la Réunion, l'élevage bovin allaitant est une composante essentielle du développement territorial. Pour assurer sa pérennité, il doit toutefois améliorer sa productivité en raison de performances moyennes et des pressions de l'environnement économique. La construction d'un conseil adapté passe par une bonne connaissance des pratiques des éleveurs. Dans cette perspective, cet article expose les résultats de suivi de dix élevages bovins naisseurs en termes de performances animales et de pratiques d'allotement, de pâturage et de complémentation.

A la Réunion, l'élevage bovin constitue un enjeu de développement économique et territorial. Le Plan d'Aménagement des Hauts, mis en place dans les années 70, a favorisé l'installation d'élevages bovins dans les Hauts afin de lutter contre l'exode rural lié à la crise du géranium. Moins connu que la canne à sucre qui est la culture emblématique des Bas, il en constitue pourtant le «pendant» pour les Hauts. L'effectif total de bovins, déclaré au recensement agricole de 2000, est de 27092 têtes réparties dans 2037 exploitations. On peut toutefois distin- guer un élevage bovin «diffus», avec la présence de quelques têtes dans de nombreuses petites exploitations agricoles, d'un élevage bovin s'inscrivant dans une agriculture conventionnelle adossée à des filières organisées. L'enquête structure 2005 recense, quant à elle, 986 «exploitations professionnelles» ayant des bovins (Agreste 2005, enquête structure). Parmi elles, les élevages naisseurs, auxquels nous nous sommes intéressés dans cette étude, représentent 8828 vaches mères pour 497 exploitations. Les autres exploitations sont com- posées d'élevages laitiers (5546 vaches pour 184 exploitations) et d'élevages engraisseurs.

La filière allaitante s'est structurée en quelques décennies selon un modèle biogéographique original. Des troupeaux naisseurs se sont installés dans les «Hauts» de l'île où ils sont conduits quasi exclusivement au pâturage. Ils sont essentiellement situés dans les Hauts de l'Ouest et à la Plaine des Cafres (figure 1). Les broutards issus de ces élevages sont vendus à des

Figure 1. Pluviométrie et température mensuelles dans trois régions de la Réunion (moyennes calculées sur les années 1999-2002). Sur la carte, la taille des cercles est proportionnelle au cheptel bovin recensé au recensement agricole 2000.

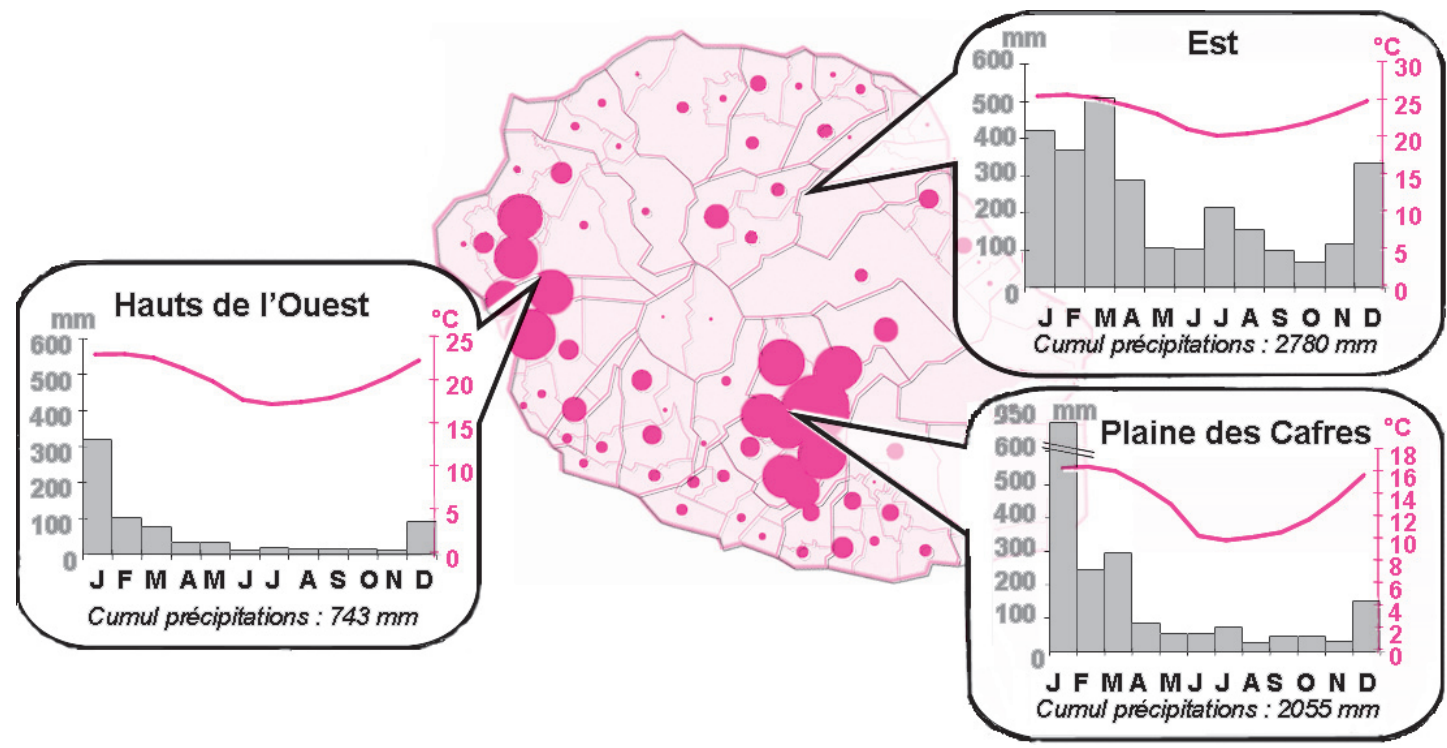


ateliers d'engraissement situés dans les «Bas», où les animaux sont finis à l'auge. Ces ateliers constituent une forme de diversification d'exploitations souvent orientées sur la production de canne à sucre.

Les filières bovines font l'objet de soutiens publics importants destinés à accompagner leur développement et à garantir la rentabilité des exploitations. Ces soutiens sont, notamment, justifiés par des coûts de production, en moyenne deux fois plus élevés qu'en métropole (Choisis et al 2003). Ces surcoûts sont liés à l'éloignement, et donc à la nécessité d'importer tous les intrants et les équipements, et à des tailles d'exploitation modestes (18 vaches mères vs 37 pour la métropole (Agreste 2005)) limitant les économies d'échelle. Ces soutiens sont toutefois subordonnés à des attentes en termes de maintien de l'emploi et d'accroissement des productions pour mieux satisfaire un marché local approvisionné à seulement $30 \%$ par les exploitations réunionnaises. Les possibilités d'agrandissement des fermes étant très réduites du fait des fortes contraintes foncières, l'augmentation de la production passe par une amélioration des performances et de la maîtrise technique des exploitations.

L’élevage «conventionnel» étant une activité récente, le métier d'éleveur se construit sur de nombreux emprunts à la métropole: importation de races «améliorées» (Limousine, Blonde d'Aquitaine...), adoption de techniques d'élevage (gestion des prairies, ensilage, insémination artificielle...), mais également sur la production de références locales. C'est pour accompagner cette production que le Cirad et l'Inra ont créé en 1987 une unité de recherches sur l'élevage bovin à la Réunion en coordination avec les organismes d'encadrement du Département

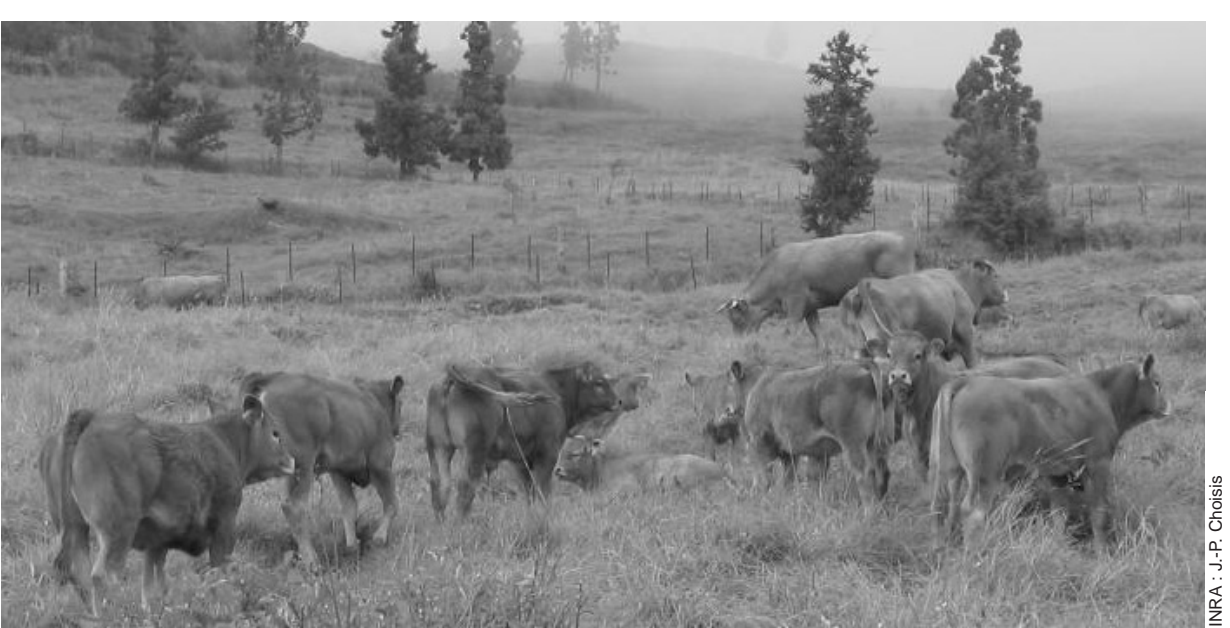

(Blanfort et al 2000). Après avoir conduit de nombreuses expérimentations sur la production et la gestion des ressources fourragères, un suivi en exploitation bovine allaitante a été mis en place afin d'étudier les pratiques mises en œuvre par les éleveurs en termes de gestion des prairies et de conduite de l'alimentation. Le rapprochement de ces pratiques avec les performances zootechniques a permis d'en identifier le lien avec la productivité des élevages (Grimaud et Thomas 2008, Choisis et al 2008), contribuant à alimenter le référentiel des «bonnes pratiques» de l'élevage bovin allaitant sur l'île de la Réunion. Un nouveau passage dans les fermes cinq années après la fin de ce suivi a permis de mesurer les changements survenus dans les exploitations. Ce sont les différentes étapes de ce travail qui sont présentées dans cet article en termes de conduite des troupeaux, de gestion des prairies et d'alimentation des animaux.

\section{1 / L'élevage bovin naisseur sur l'île de la Réunion}

En élevage naisseur, les animaux sont conduits au pâturage toute l'année. La disponibilité des ressources fourragères au pâturage varie fortement au cours de l'année avec une période d'excédent en saison des pluies (décembre à mars) et une période de déficit en saison sèche (avril à novembre). Cette alternance s'exprime également de manière très contrastée selon les régions avec des extrêmes entre l'Est, où la pluviométrie est très importante, et les Hauts de l'Ouest où le climat est beaucoup plus sec et où la pousse de l'herbe en saison fraîche est très réduite (figure 1 ). Ces différences locales sont accentuées par le gradient altitudinal, les pâturages étant composés de graminées tropicales aux altitudes les plus basses et de four- rages tempérés aux altitudes les plus hautes. Le kikuyu (Pennisetum clandestinum) prédomine dans les systèmes prairiaux jusqu'à $1200 \mathrm{~m}$; au-delà, il est associé à des graminées tempérées, telles que le ray-grass (Lolium perenne) ou le dactyle (Dactylis glomerata) qui deviennent dominantes aux altitudes les plus hautes. Enfin, on rencontre d'autres graminées tropicales dans les Bas comme le setaria (Setaria anceps), le chloris (Chloris gayana) et les cannes fourragères (Pennisetum purpureum et Tripsacum laxum) (Grimaud et Thomas 2002, Grimaud et al 2003). Les éleveurs cherchent à compenser les déficits fourragers de saison fraîche par des pratiques de complémentation avec des aliments concentrés achetés ou des sous-produits agro-industriels provenant de la culture et de la transformation de la canne à sucre (paille de canne, bagasse, mélasse). La constitution de stocks de fourrages s'est également développée sous forme d'ensilage d’herbe enrubanné. Le cheptel métissé, du fait des nombreuses races introduites, a fait l'objet d'une politique volontariste d'amélioration génétique avec l'importation de reproducteurs et de semence, majoritairement des races Limousine et Blonde d'Aquitaine. Les taureaux sont généralement conduits avec les vaches toute l'année, permettant un étalement relatif des mises bas. L'introduction de génisses prêtes à vêler de France métropolitaine a longtemps compensé une saisonnalité naturelle des naissances, liée aux variations climatiques, qui a son pic en saison fraîche (Grimaud et Thomas 2008). Les broutards restent au pâturage avec leur mère jusqu'à leur vente à un âge moyen de 9-10 mois. Globalement, les performances techniques moyennes des élevages naisseurs apparaissent encore très en deçà de leurs homologues métropolitains. Une enquête réalisée en 2001 auprès de 32 élevages naisseurs a abouti au calcul d'une productivité pondérale moyenne comprise entre 180 et $270 \mathrm{~kg}$ de viande vive produite annuellement par vache présente, selon les types d'exploitation (Choisis et al 2003). A titre de comparaison, les réseaux d'élevage français atteignent des valeurs moyennes supérieures à $360 \mathrm{~kg}$ par vache et par an (Institut de l'élevage 2005). Si les contraintes de milieu particulières de la Réunion (climat, valeur alimentaire des fourrages tropicaux, parasitisme et maladies tropicales) peuvent expliquer en partie ce faible niveau de productivité, on observe des différences notables entre exploitations qui laissent augurer des marges de progrès encore importantes. 
Tableau 1. Principales caractéristiques des dix élevages suivis.

\begin{tabular}{|c|c|c|c|c|c|c|}
\hline Eleveur & Localisation & Activité & $\begin{array}{c}\text { SFP } \\
\text { (ha) }\end{array}$ & $\begin{array}{c}\text { Nombre de } \\
\text { vaches }\end{array}$ & $\begin{array}{c}\text { Nombre } \\
\text { d'UGB }\end{array}$ & $\begin{array}{c}\text { Chargement } \\
\text { (UGB/ha) }\end{array}$ \\
\hline 1 & Est & Naisseur -Engraisseur & 37 & 62 & 91 & 2,46 \\
2 & Plaine des Cafres & Naisseur- Engraisseur & 72 & 62 & 87 & 1,21 \\
3 & Hauts de l'Ouest & Naisseur & 60 & 55 & 60 & 1,01 \\
4 & Plaine des Cafres & Naisseur & 38 & 48 & 59 & 1,55 \\
5 & Hauts de l'Ouest & Naisseur & 25 & 53 & 64 & 2,55 \\
6 & Hauts de l'Ouest & Naisseur & 29 & 45 & 50 & 1,72 \\
7 & Hauts de l'Ouest & Naisseur & 75 & 52 & 64 & 0,85 \\
8 & Plaine des Cafres & Naisseur & 66 & 42 & 52 & 0,79 \\
9 & Plaine des Cafres & Naisseur & 55 & 69 & 82 & 1,49 \\
10 & Plaine des Cafres & Naisseur-Sélectionneur & 60 & 53 & 62 & 1,05 \\
\hline \multicolumn{2}{|r|}{ Moyenne } & 52 & 54 & 67 & 1,47 \\
\hline
\end{tabular}

Dans cette perspective, la construction d'un conseil adapté à la diversité des situations doit reposer sur une connaissance précise des pratiques mises en œuvre par les éleveurs et des résultats techniques et économiques.

\section{2 / Modalités de conduite du suivi}

\section{1 / Description des exploita- tions}

Dix élevages naisseurs situés dans les Hauts de la Réunion ont fait l’objet d'un suivi de 1999 à 2002. Cinq sont situés à la Plaine des Cafres, quatre dans les Hauts de l'Ouest et un dans l'Est. Ces trois régions aux caractéristiques climatiques très différentes induisent une gestion particulière des systèmes fourragers. Les éleveurs doi- vent ainsi tenir compte de contraintes régionales, comme le parasitisme et le piétinement dans l'Est ou l'impact de la sécheresse dans l'Ouest. Toutes les exploitations sont des élevages naisseurs, dont le système d'alimentation est fondé sur le pâturage. L'échantillon ne compte que des exploitations de taille moyenne à grande, relativement spécialisées dans l'élevage bovin. Elles disposent d'une Surface Fourragère Principale (SFP) comprise entre 25 et 75 ha et le nombre de vaches allaitantes par exploitation varie de 42 à 69 têtes (tableau 1). La constitution de l'échantillon a tenu compte de la représentativité géographique et de l'inscription des exploitations dans des réseaux techniques. En conséquence, ce sont des exploitations assez suivies dont les performances sont globalement supérieures à la moyenne des éleveurs adhérents à la SicaRévia (SICA Réunion Viande). Le tableau 2 permet de situer

Tableau 2. Performances moyennes d'élevages allaitants inscrits au contrôle de performances.

\begin{tabular}{|l|c|}
\hline Nombre d'exploitations & 77 \\
Nombre moyen de vaches & 43 \\
Veaux nés par vache & 0,78 \\
Intervalle vêlage-vêlage (j) & 440 \\
\hline Source : EDE (année 2002)
\end{tabular}

\begin{tabular}{|l|c|c|}
\hline & Moyenne & $\boldsymbol{n}$ \\
\hline PAT 120j - mâles (kg) & 139 & 952 \\
PAT 120j - femelles (kg) & 129 & 888 \\
\hline
\end{tabular}

PAT : Poids à Age Type

Source : EDE (année 1999) ces exploitations vis-à-vis de quelques critères de performances (à comparer aux données des tableaux 1 et 4).

\section{2 / Collecte des données}

Le suivi des exploitations a reposé sur un passage trimestriel dans les élevages où ont été recueillies des données relatives aux performances zootechniques de l'exploitation (pesée des broutards et notation d'état corporel des vaches allaitantes), à l'état de santé du troupeau (prises de sang et coproscopies), et à l'observation des parcelles pâturées (mesures de hauteur d'herbe). Ces données étaient complétées à chaque passage par les inventaires et les mouvements d'animaux fournis par les éleveurs, et confirmés par l'EDE. L'analyse des pratiques s'est fondée sur un entretien avec l'éleveur autour d'un calendrier de pâturage réactualisé lors de chaque visite et prenant en compte la fertilisation des prairies, le rythme et l'ordre de rotation sur les parcelles, et la complémentation distribuée par lot d'animaux.

A partir de ces données, des paramètres ont été calculés pour évaluer :

- les pratiques : biovolumes d'herbe, temps de présence des animaux, temps de repos des parcelles, chargement animal, complémentation, taux de réforme, etc. (tableau 3),

- les performances animales : gains moyens quotidiens, poids à âge type,

Tableau 3. Moyennes et dispersion de quelques indicateurs de pratiques des élevages suivis.

\begin{tabular}{|l|c|c|c|c|}
\hline \multicolumn{1}{|c|}{ Variable } & Moyenne & $\begin{array}{c}\text { Ecart- } \\
\text { type }\end{array}$ & Min & Max \\
\hline Durée moyenne du pâturage (j/ha) & 2,8 & 1,8 & 1,1 & 5,4 \\
Taille moyenne des parcelles (ha) & 8,0 & 8,8 & 0,8 & 29,4 \\
Temps d'interpassage moyen de la rotation (j) & 74,5 & 35,9 & 37,5 & 148 \\
Chargement (UGB/ha de SFP) & 1,5 & 0,6 & 0,8 & 2,6 \\
Complémentation des broutards (UF/an) & 434,2 & 270 & 0 & 857,5 \\
Complémentation des vaches allaitantes (UF/an) & 741,9 & 429,7 & 46,0 & 1527,1 \\
Taux de réforme (\%) & 11,3 & 4,4 & 4,7 & 17,7 \\
\hline
\end{tabular}


Tableau 4. Moyennes et dispersion de quelques indicateurs de performances des élevages suivis.

\begin{tabular}{|l|c|c|c|c|c|c|}
\hline & Moyenne & $\begin{array}{c}\text { Ecart- } \\
\text { type }\end{array}$ & $\begin{array}{c}\text { CV } \\
(\%)\end{array}$ & Min & Max & $\boldsymbol{n}$ \\
\hline Taux de vêlages (\%) & 80,6 & 13,0 & 16,1 & 58,3 & 94,8 & \\
Intervalle Vêlage-Vêlage (j) & 383 & 30,2 & 7,9 & 353,9 & 442,4 & 1097 \\
Age au premier vêlage (j) & 1052,1 & 68,2 & 6,5 & 973,7 & 1207,3 & 319 \\
\hline PAT 120j - mâles $(\mathrm{kg})$ & 148,6 & 21,6 & 14,5 & 119,9 & 180,5 & 747 \\
PAT120j-femelles $(\mathrm{kg})$ & 137,4 & 18,1 & 13,2 & 111,3 & 166,1 & 740 \\
PAT 210j - mâles $(\mathrm{kg})$ & 227,9 & 39,1 & 17,2 & 171,9 & 283,5 & 772 \\
PAT 210j - femelles $(\mathrm{kg})$ & 207,9 & 31,4 & 15,1 & 161,9 & 252,4 & 765 \\
\hline Mortalité des veaux (\%) & 11,2 & 3,7 & 33,0 & 4,2 & 16,2 & \\
PBVV/ha $(\mathrm{kg})$ & 274,8 & 92,0 & 33,5 & 150,3 & 397,9 & \\
PBVV/vache $(\mathrm{kg})$ & 239,9 & 61,5 & 25,6 & 170,7 & 316 & \\
\hline
\end{tabular}

intervalle vêlage-vêlage, taux de mise bas, taux de mortalité, Production Bovine de Viande Vive (PBVV) (tableau 4). Ce dernier paramètre, utilisé dans les réseaux d'élevage (Sarzeaud 2002), permet de disposer d'une vision synthétique des performances de l'exploitation. Il est basé, ici, sur l'estimation de la production de viande vive des animaux de moins de un an au cours de l'année, calculée à partir des courbes de croissance. Il prend en compte la variation de poids des jeunes présents entre la fin et le début de l'année et le poids des broutards vendus.
Ces données ont été enregistrées dans la base de données SEBARUN (Suivi des Elevages Bovins Allaitants de la RéUNion), permettant le calcul et la restitution aux éleveurs d'indicateurs de performances au niveau de l'animal et de la parcelle (Grimaud et Thomas 2008).

Un nouvel entretien a eu lieu dans chacune de ces exploitations cinq années après la fin de ce suivi. Une discussion avec l'éleveur conduite sur la base des différents indicateurs calculés a été l'occasion de l'interroger sur ses stratégies et l'évolution de ses pratiques depuis l'arrêt du suivi.

\section{3 / Résultats}

\section{1 / Allotement des animaux}

Les observations conduites en 2000 mettent en évidence des pratiques d'allotement variables selon les éleveurs (figure 2). Nous distinguons ainsi la constitution de lots principaux de vaches mères et de lots secondaires. Au cours du suivi, le nombre de lots principaux dépend de considérations liées à la taille du troupeau, à celle des parcelles, à des choix en matière de reproduction et à une volonté de

Figure 2. Schéma récapitulatif des contraintes et des choix qui justifient les pratiques d'allotement (en noir : 2000, en rouge : 2006, souligné : 2000 et 2006).

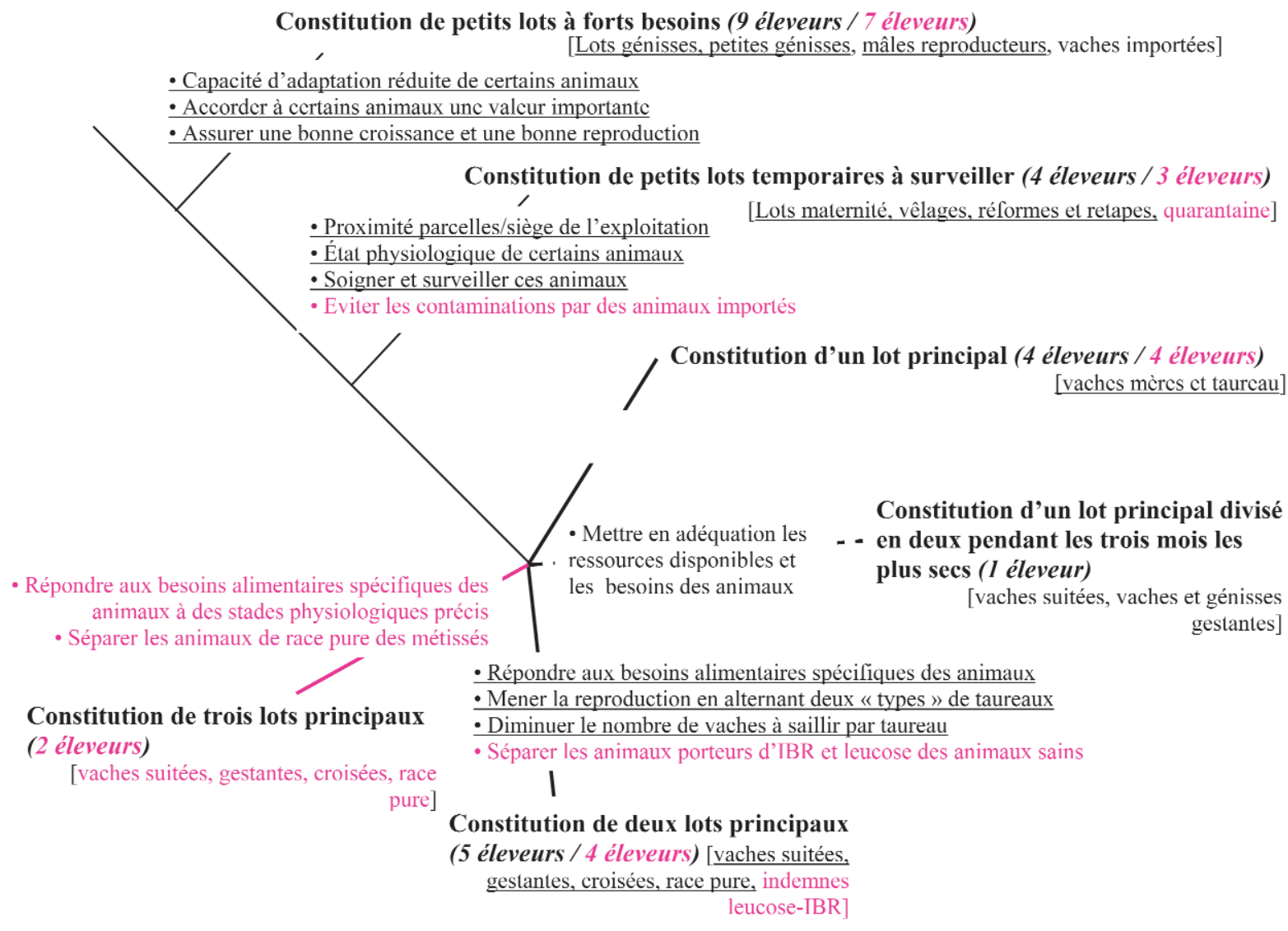


Figure 3. Schéma récapitulatif des pratiques de gestion des prairies observées et de leur évolution entre 2000 et 2006 (les effectifs ne peuvent être additionnés car quelques éleveurs adoptent des conduites différentes selon les lots).

Figure 3a. Saison humide

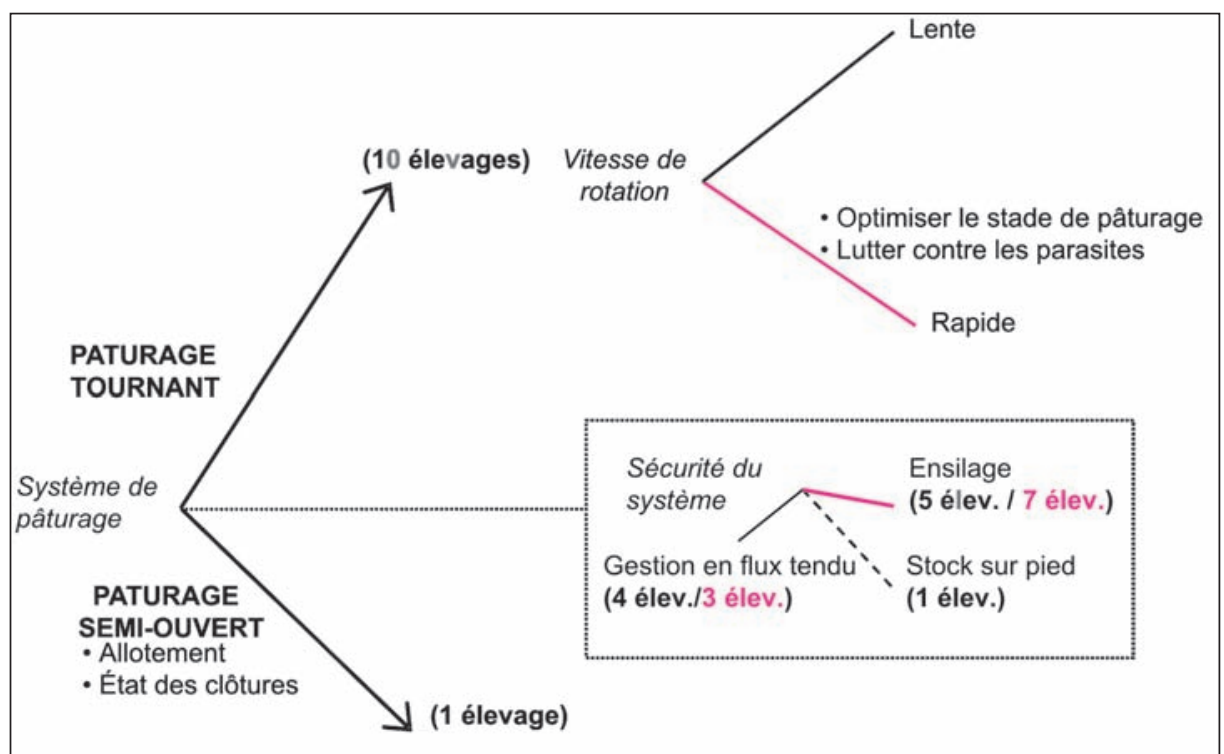

Figure 3b. Saison sèche

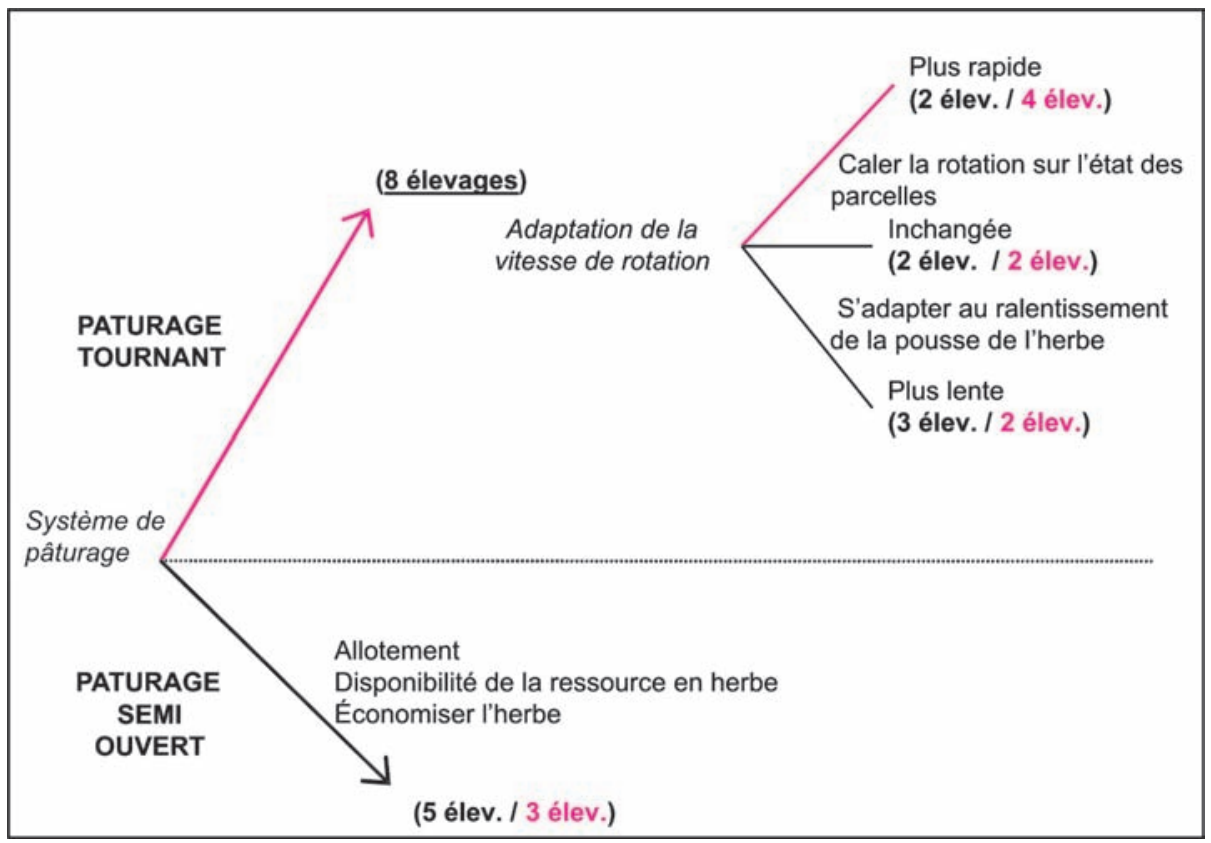

complémenter différemment plusieurs catégories d'animaux (par exemple, les vaches suitées vs les autres vaches). Dans 9 cas sur 10, des lots secondaires sont également constitués pour permettre une conduite adaptée à des catégories d'animaux. Il s’agit généralement de lots de génisses dont on veut assurer une bonne croissance et une bonne reproduction et qui seront inséminées. Pour les mêmes raisons, les vaches importées sont également conduites séparément. Certains éleveurs importent en effet des génisses ou des jeunes vaches dans la perspective de vendre l'éleveur cherchera à améliorer leur état corporel avant la vente.

Entre 2000 et 2006, tous les élevages ont eu des changements dans leurs pratiques d'allotement (figure 2). Deux éleveurs ont simplifié leur conduite. Le premier, qui scindait son troupeau en deux pendant la saison sèche (vaches suitées vs vaches et génisses gestantes), ne le faisait plus en 2006. Son choix est de vendre les broutards et les vaches de réforme avant la saison sèche de façon à réduire le chargement et la complémentation. Le second éleveur, dans une perspective de départ à la retraite, a rassemblé les trois lots antérieurs en un seul troupeau. A contrario, trois éleveurs ont augmenté le nombre de lots principaux. Un éleveur est passé de un à deux, et les deux autres éleveurs de deux à trois. Cet accroissement du nombre de lots provient soit de la volonté de séparer plus finement les animaux de stade physiologique différent pour adapter leur alimentation, soit de la croissance du lot d'animaux de race pure qui est ainsi devenu un lot principal. Par ailleurs, de nouvelles considérations d'ordre sanitaire sont apparues avec la mise en œuvre du plan d'éradication de la leucose bovine et de l'IBR (Infectious Bovine Rhinotracheitis) qui ont conduit trois éleveurs à séparer les animaux séropositifs et indemnes ou à constituer un lot de quarantaine.

\section{2 / Gestion du pâturage}

Les éleveurs sont confrontés à l'alternance de périodes excédentaires, où la croissance rapide de l'herbe ne leur permet pas de la faire pâturer avant qu'elle ne perde en qualité, et de périodes de sécheresse où l'herbe seule ne suffit pas à nourrir les animaux. La figure 3 montre, pour ces deux saisons, les différentes pratiques mises en œuvre par les éleveurs et leur évolution. Tous les éleveurs pratiquent le pâturage tournant en saison chaude et humide, un seul pratiquant aussi le pâturage semi-ouvert, qui consiste à laisser pâturer conjointement plusieurs parcelles à cette période. Le rythme de rotation varie d'une exploitation à l'autre dans une amplitude de durée de présence des animaux sur la parcelle qui va de 3 à 12 jours. Les rythmes plus rapides sont associés à la volonté «d'optimiser la qualité de l'herbe», vs les rythmes plus lents, pour lesquels les éleveurs expriment le besoin de «voir de l'herbe». Ils font indirectement la relation entre volumes disponibles et satisfaction des besoins des animaux. En saison fraiche et sèche, la majorité 


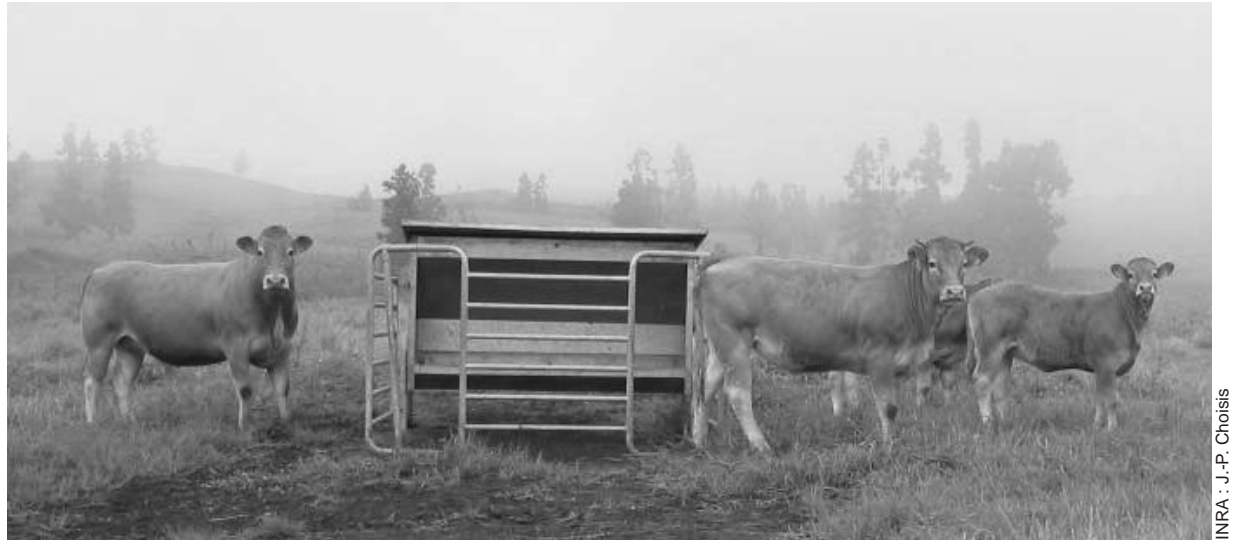

des éleveurs maintiennent le pâturage tournant, mais adaptent sa vitesse de rotation ; cinq éleveurs, dont tous ceux des Hauts de l'Ouest, choisissent en partie le pâturage semi-ouvert. Ils le justifient par une sécheresse trop marquée pour autoriser le pâturage tournant. Parfois, ils réservent le pâturage semi-ouvert au lot principal constitué des animaux dont les besoins sont plus faibles (vaches non suitées, vaches croisées), conscients que cette pratique ne permet pas une bonne exploitation de l'herbe sur le plan qualitatif.

D’autres considérations peuvent être mises en avant pour justifier de l'adoption d'un mode de conduite, tels que la lutte contre les endoparasites pour le pâturage tournant ou le mauvais état des clôtures pour le pâturage semiouvert. Des huit éleveurs qui conservent le pâturage tournant toute l'année, trois adaptent le rythme de rotation à la pousse de l'herbe, qui est donc plus lent en saison sèche. Deux éleveurs adoptent, a contrario, un rythme plus rapide en saison sèche car, selon l'expression des éleveurs, les animaux «demandent lorsque la ressource manque. Deux éleveurs, enfin, ne changent pas de rythme car ils ont un chargement faible qui leur assure une quantité d'herbe suffisante toute l'année et ils peuvent, par conséquent, suivre un rythme de rotation très rapide (2 ou 3 j par parcelle). Ces pratiques ont été peu modifiées entre 2000 et 2006, hormis l'augmentation de la vitesse de rotation en saison sèche pour deux éleveurs (figure 3).

Les éleveurs cherchent à sécuriser le système de manière à réduire l'impact de ces variations saisonnières. L'ajustement du rythme de rotation au pâturage, de façon à consommer rapidement la ressource disponible, constitue une réponse que nous avons qualifiée de «flux tendus». Mais les éleveurs constituent également des stocks, pendant la saison des pluies, qu'ils exploitent pendant la saison sèche. Un éleveur pratiquait ainsi, en 2000, le stock d'herbe sur pied, qui consiste à mettre une parcelle en défens en fin de saison à changer» plus rapidement de parcelle humide pour constituer une réserve qui sera pâturée en saison sèche. Cette pratique présente cependant le défaut majeur de fournir une ressource de très mauvaise qualité. Pour conserver la qualité du fourrage, une solution de plus en plus répandue est la réalisation d'ensilage d'herbe enrubanné. Cette technique largement adoptée par les éleveurs laitiers a été, avec l'accroissement de la taille des troupeaux, progressivement mise en œuvre par les éleveurs allaitants de la Plaine des Cafres puis par ceux des Hauts de l'Ouest. A l'échelle de la Réunion, de nombreux éleveurs ont ainsi investi dans des chaînes d'ensilage. Dans le cadre du suivi, ils étaient cinq à le pratiquer déjà en 2000 , et sept en 2006, alors que plus aucun éleveur ne pratiquait le stock sur pied.

\section{3 / Complémentation des ani- maux}

Les pratiques de complémentation concernent l'apport de concentré du commerce, de sous-produits de la canne à sucre (mélasse, bagasse, paille de canne), et de fourrages conservés achetés ou produits sur l'exploitation (foin de Chloris gayana et ensilage d'herbe). Tous compléments confondus, les apports moyens par tête montrent une forte variabilité entre les élevages (tableau 3). Elle résulte d'une variété de pratiques, plus importante pour les femelles que pour les mâles, qui traduit le souci d'adapter les apports aux besoins physiologiques et à la saison. Pour disposer d'une représentation synthétique, nous les avons projetées sur un chronogramme qui situe les grandes catégories d'animaux (figure 4).

Figure 4. Schéma récapitulatif des pratiques de complémentation. Les justifications (en rouge) sont positionnées sur un axe de temps lié à la carrière des mâles (axe du haut) et des femelles (axe du bas) (SH : saison humide, SS : saison sèche).

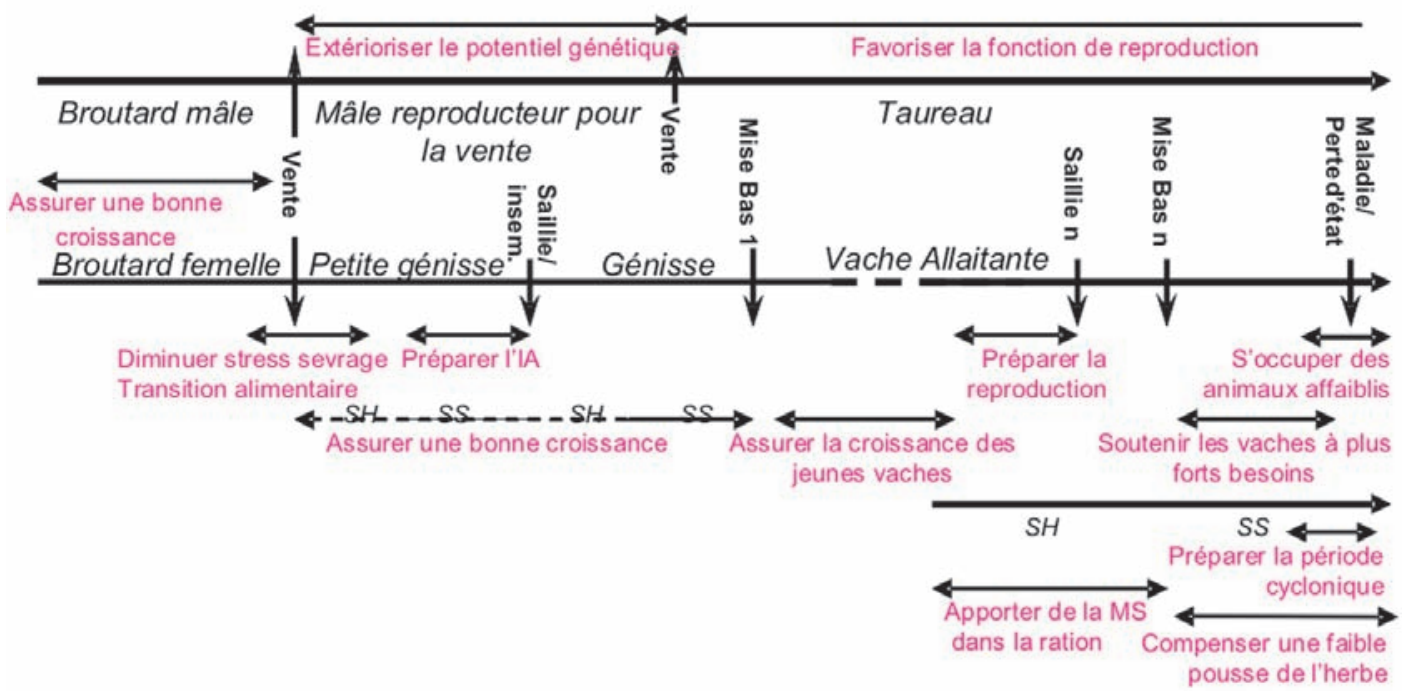


Tableau 5. Matrice de corrélations des indicateurs de performances des élevages suivis.

\begin{tabular}{|l|c|c|c|c|c|}
\hline & $\begin{array}{c}\text { Age } \\
1^{\text {er vêlage }}\end{array}$ & MB & IVV & P120M & Mortalité \\
\hline Taux de vêlages (MB) & $-0,83^{* *}$ & & & & \\
Intervalle Vêlage-Vêlage & 0,53 & $-0,88^{* * *}$ & & & \\
(IVV) & $-0,65^{*}$ & $0,91^{* * *}$ & $-0,84^{* *}$ & & \\
Poids à 120j des mâles & $-0,12$ & $-0,33$ & 0,57 & $-0,47$ & \\
(P120M) & $-0,63^{*}$ & $0,89^{* * *}$ & $-0,85^{* *}$ & $0,94^{* * *}$ & $-0,49$ \\
Mortalité des veaux (\%) & & \\
PBVV/vache &
\end{tabular}

${ }^{*} \mathrm{P}<0,05{ }^{* *} \mathrm{P}<0,01{ }^{* * *} \mathrm{P}<0,001$.

Figure 5. Relation entre le taux de vêlage et le poids à âge-type à 120 jours des élevages suivis.

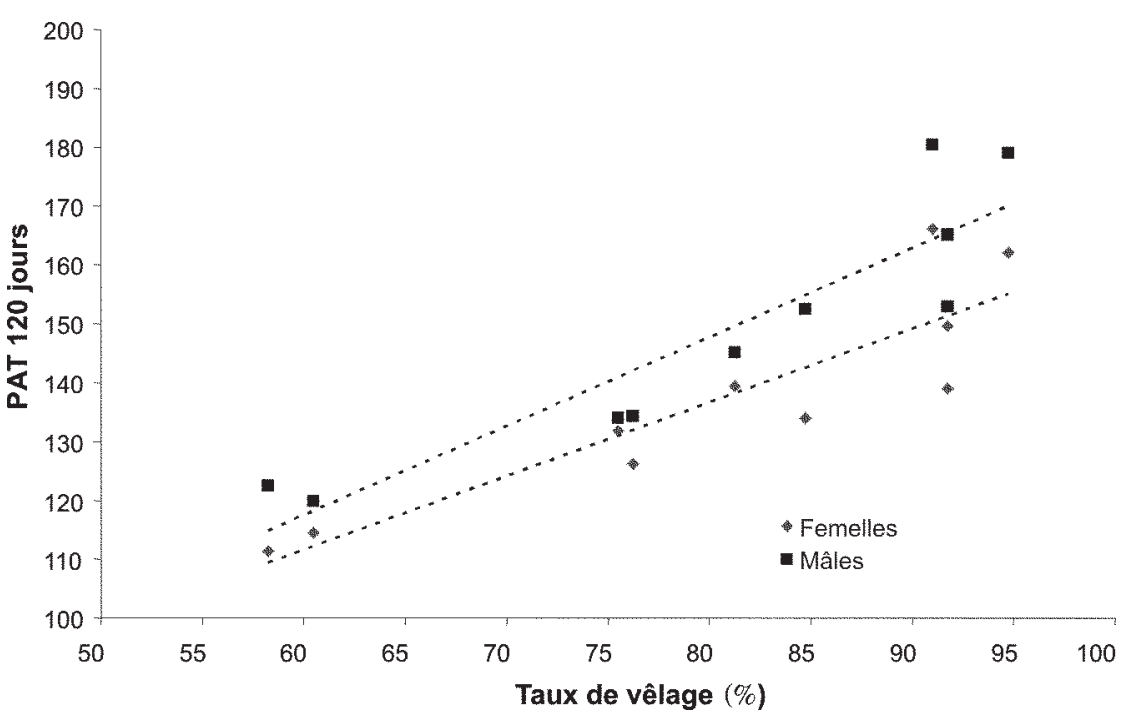

Figure 6. Relation entre chargement et production de viande vive pour l'ensemble des couples éleveur x année (en légende : niveau de complémentation des vaches).

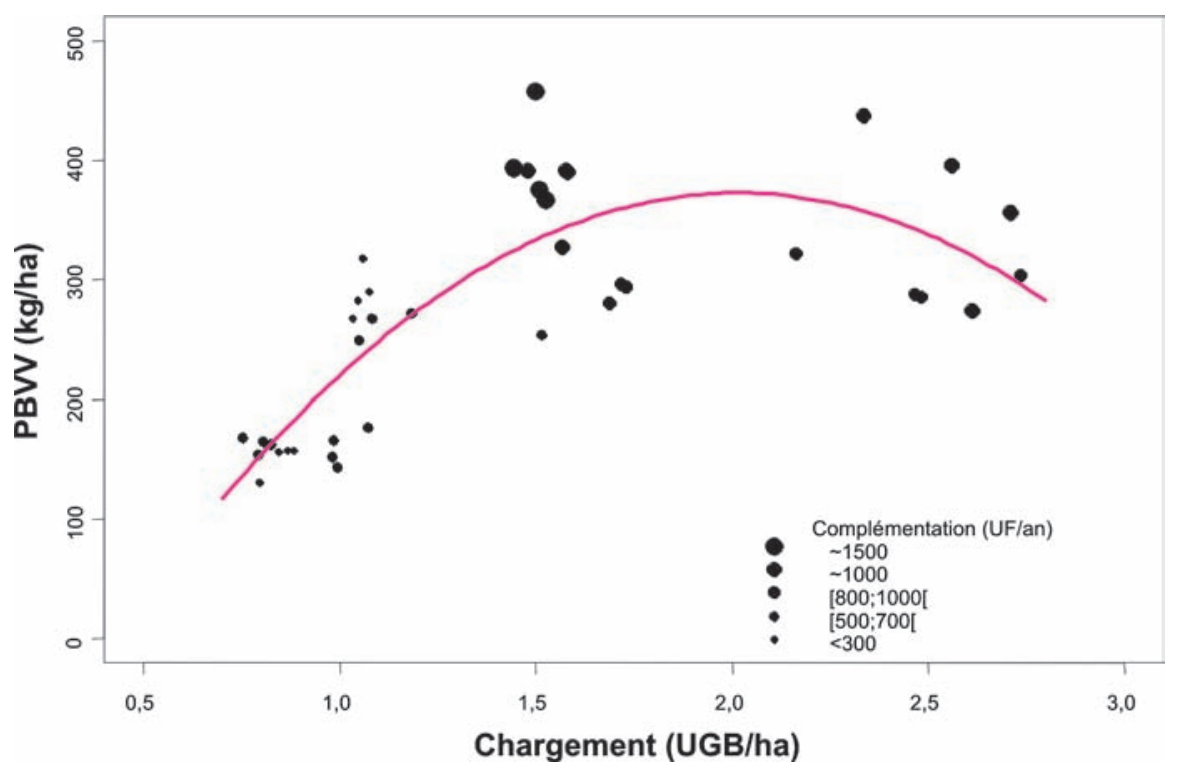

Les mâles reproducteurs destinés à la vente étaient systématiquement complémentés afin d'extérioriser le patrimoine génétique des animaux. De tout comme la complémentation des broutards au nourrisseur qui est mise en œuvre pour assurer une bonne croissance (seul un éleveur ne complémente pas ses broutards).

Par contre, la complémentation des femelles de renouvellement est plus liée à des choix individuels. Si les génisses sont généralement complémentées, elles peuvent l'être soit toute l'année, soit uniquement en saison sèche afin de soutenir leur croissance. Elle est parfois pratiquée ponctuellement dans le but de réduire le stress lié au sevrage, ou à un changement de lot, en assurant ainsi une transition alimentaire. Certains éleveurs distribuent également du concentré en préparation de l'insémination afin d'obtenir de meilleures performances de reproduction.

Quelques éleveurs complémentent les vaches allaitantes jusqu'à leur 3 ème mise bas, pour assurer leur croissance mais, dans la plupart des cas, les vaches ne font pas l'objet d'une complémentation permanente. Des apports peuvent être effectués pendant les 3 ou 4 mois qui suivent la mise bas, tout au long de l'année et, plus généralement, pendant les trois mois les plus secs. Il s'agit alors d'aliments grossiers (paille de canne, bagasse, ensilage) et/ou d'aliment concentré.

L'éleveur situé dans l'Est se distingue par une complémentation originale en saison humide destinée à apporter de la matière sèche dans la ration : il apporte des aliments concentrés à ses animaux afin de limiter les diarrhées liées à la forte teneur en eau de l'herbe pâturée.

L'enquête menée en 2006 indique que les déterminants des pratiques de complémentation exposés sur la figure 4 sont toujours valides. On note, cependant, que chaque éleveur a ajusté ses pratiques de complémentation au milieu, aux pratiques d'allotement et de gestion des ressources fourragères, de telle sorte que chacun a créé un système de complémentation qui lui est propre. Complémentation et allotement sont combinés de manière à être en adéquation avec le stade physiologique des animaux, la période de l'année et les terrains pâturés par chaque lot.

\section{4 / Performances zootech- niques}

Malgré la dimension réduite de l'échantillon, l'analyse de variance révèle une différence très hautement 


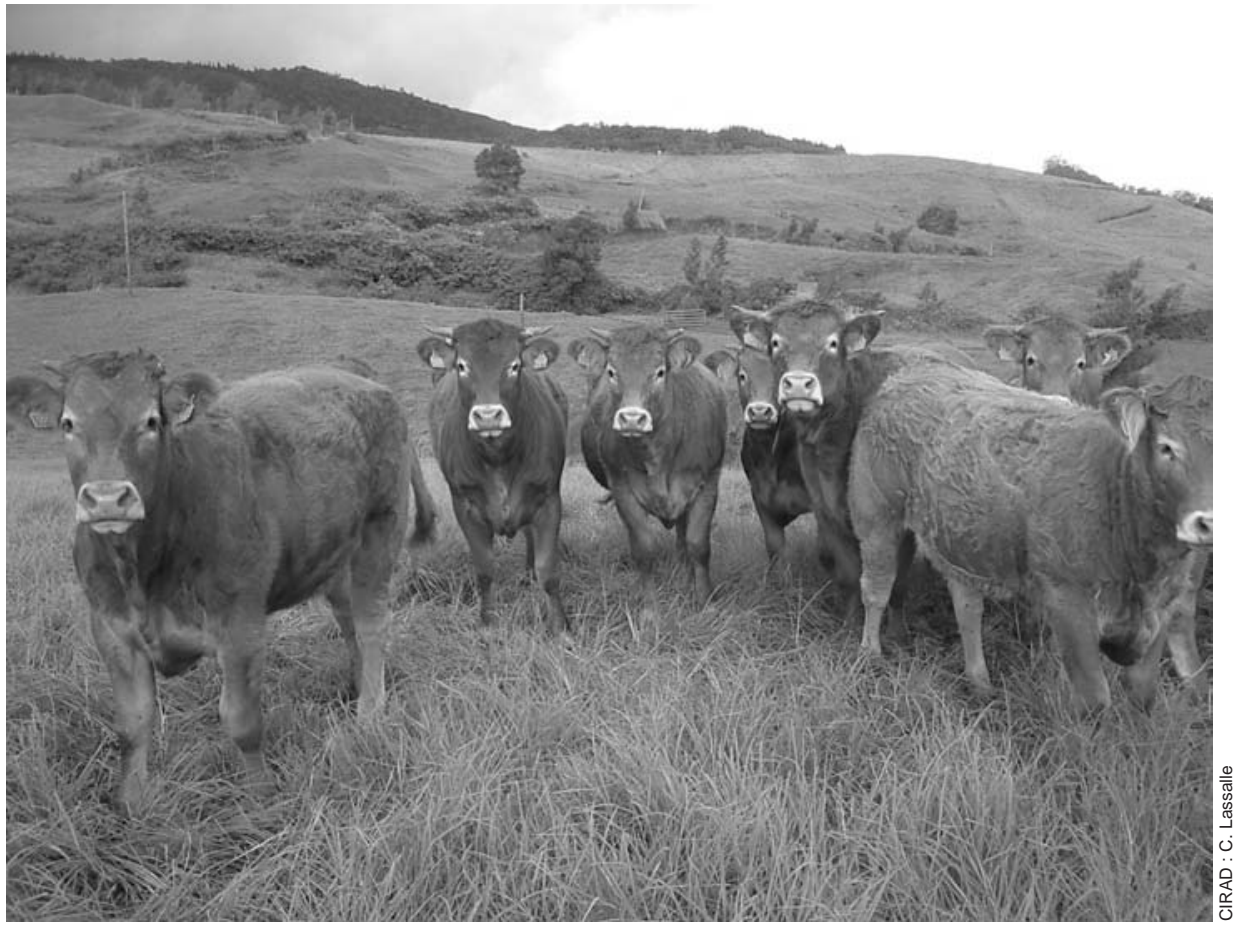

significative entre exploitations pour toutes les performances zootechniques individuelles (âge au premier vêlage, intervalle entre vêlages, poids à âge type, tableau 4). La dispersion relative est élevée pour la mortalité des veaux puis, de manière équivalente, pour le taux de vêlage et les poids à âge-type. On observe, par ailleurs, que les critères de reproduction et de croissance sont fortement corrélés (tableau 5), comme le montre la relation entre les taux de vêlages et les Poids à Age Type (PAT) 120 jours moyens pour les dix exploitations (figure 5). La Production Brute de viande Vive (PBVV), calculée par vache, constitue donc un indicateur synthétique pertinent pour évaluer la performance globale du troupeau. Pour évaluer la performance globale de l'exploitation, la PBVV doit être rapportée à l'unité de surface fourragère. Celle-ci s'accroît avec le chargement jusqu'au seuil de 2 Unités Gros Bovins (UGB)/ha, avant de diminuer (figure 6). La complémentation des vaches compense l'augmentation du chargement et permet d'obtenir une productivité plus élevée (on notera que les éleveurs qui ont un niveau de chargement proche d'une UGB complémentent très peu leurs vaches).

\section{4 / Discussion et conclusion}

Les exploitations suivies sont représentatives des élevages naisseurs des Hauts de la Réunion malgré le biais introduit dans leur choix et nous semblent bien éclairer la diversité des conduites mises en œuvre. On les retrouve, en effet, dans une majorité des dix types d'élevages naisseurs et naisseurs-engraisseurs identifiés dans le cadre d'une enquête réalisée auprès des éleveurs adhérents de la coopérative (Lacroix 2002). Les petites exploitations diversifiées, dont la productivité est plus faible, ne sont toutefois pas représentées dans l'échantillon. De même, l'élevage diffus qui est également présent dans les Bas n'a pas été pris en compte dans cette étude. Il correspond à une autre logique de production, proche de ce que l'on observe en Guadeloupe (Diman et al 2002). Ce sont des élevages de quelques têtes, localisés dans des exploitations de petite dimension, qui se positionnent plutôt sur un marché informel. Cette diversité qui est souvent pointée dans les typologies d'exploitation est accentuée à la Réunion par les différences liées au climat et à l'altitude. Chaque éleveur doit donc faire face à des conditions pédoclimatiques et topographiques différentes, et l'analyse multivariée, conduite par ailleurs, portant sur les relations entre les pratiques mises en œuvre et les performances correspondantes n'a pas permis de regrouper les éleveurs en un nombre réduit de classes typologiques (Choisis et al 2008).

Pour atteindre leurs objectifs de production, les éleveurs vont essayer d'ajuster la dimension des parcelles, la constitution des lots, le chargement et les apports alimentaires selon une combinaison propre à chaque exploitation.
L’éloignement de la métropole et la forte présence de l'appareil de recherche et de développement, rapportée au nombre d'éleveurs, peuvent laisser penser que l'on se trouve face à une situation atypique. En réalité, ici comme ailleurs, les agriculteurs ne font pas qu'adopter ou non les innovations proposées par les techniciens, mais les adaptent et les transforment (Figuié 2001).

Les savoirs des éleveurs réunionnais sont donc composites et résultent d'informations issues de leurs pairs métropolitains (des voyages d'étude réguliers sont organisés), des réseaux d'éleveurs locaux, de leur expérience personnelle et des conseils apportés par les techniciens. Magne (2007) parle d'activité informationnelle pour désigner un processus qui est intrinsèque à chaque individu. Ces ressources informationnelles variées sont donc intégrées dans des décisions d'action qui sont mises à l'épreuve et révisées au cours du temps. Pour les élevages suivis, on observe ainsi une évolution entre 2000 et 2006 qui traduit une adaptation des pratiques aux besoins des animaux (nutritionnels, sanitaires), à l'objectif de production des éleveurs et aux recommandations des techniciens. Pour que ces dernières soient pertinentes, il est nécessaire d'accéder à une bonne connaissance des pratiques et des projets des éleveurs (Vivier 1990). Dans cette perspective, nous discuterons les pratiques d'allotement et de conduite du pâturage dans cette dernière partie.

Les pratiques d'allotement, pour lesquelles il n'existe pas de normes explicites, sont le fruit d'une démarche personnelle enrichie par l'expérience d'autres éleveurs géographiquement ou socialement proches. Pour autant, ces pratiques ont des similitudes avec celles mises en œuvre dans les élevages métropolitains. On retrouve ainsi les principaux critères d'allotement identifiés en troupeaux bovins allaitants limousins et charolais : la taille du lot, la date de vêlage, la génétique, la réforme (Ingrand et al 1999). Sur les exploitations suivies, la recherche d'une plus grande homogénéité des lots, du point de vue du stade physiologique et des besoins nutritionnels, semble être un des objectifs majeurs de l'allotement, contrairement aux élevages métropolitains précédemment évoqués pour lesquels elle ne représenterait qu'un tiers des lots de vaches à l'échelle de la campagne (ibid). Une différence majeure réside dans le fait que l'allotement n'est pas rythmé par les cycles de mise à 
l'herbe et de rentrée à l'étable. A la Réunion, les vaches sont au pâturage et mettent bas tout au long de l'année, ce qui peut justifier l'existence de lots qui ont une relative permanence dans le temps mais dont les éléments qui les constituent se renouvellent fréquemment. Les lots de vaches et de jeunes animaux constitués ont souvent pour objectif de leur assurer une alimentation spécifique, y compris lorsque le critère d'allotement est également de nature génétique. Les génisses et vaches importées sont considérées comme des animaux plus fragiles qui requièrent des apports alimentaires supérieurs aux animaux croisés. Leur gestion dans un lot séparé permet de les complémenter ou de leur donner accès à des ressources pâturées de meilleure qualité. Si les lots ont été relativement stables en cours de campagne, ils ont, par contre, changé au cours de la période observée, sous l’influence, d'une part, de l'accroissement de la taille du troupeau et, d'autre part, de l'apparition d'un nouveau critère d'allotement avec la mise en place du plan de lutte sanitaire.

En ce qui concerne la conduite du pâturage, il est instructif de constater que des modalités de conduite très similaires ont été observées dans un contexte sensiblement différent. Hostiou et al (2006) distinguent des modalités qui visent à i) ajuster les rythmes de pâturage à la saison ; l'objectif étant de faire consommer l'herbe au meilleur stade, ii) ajuster la surface à la saison, en ouvrant les barrières en saison sèche (ce que nous avons appelé le pâturage semi ouvert), et iii) satisfai- re les besoins des vaches toute l'année en ayant une ressource en excédent, ce qui conduit à un faible taux d'exploitation de l'herbe.

Ces modalités correspondent à des logiques de production différentes que l'on retrouve sur les élevages suivis. Ces auteurs opposent des logiques de productivité des surfaces et des troupeaux au maintien du troupeau à moindre coût. Dans notre cas, l'ajustement des rythmes de pâturage à la saison, qui s'accorde avec les recommandations faites par les conseillers, se retrouve chez les éleveurs qui cherchent à maximiser les performances individuelles. Ils visent l'expression du potentiel génétique dans la perspective de vendre une partie importante des broutards, mâles et femelles, pour la reproduction à des prix plus élevés. Ce choix se traduit par une forte contrainte de travail liée aux changements fréquents de parcelles et au nombre de lots présents. C'est une conduite que nous avons qualifiée de «flux tendus». Elle s’accompagne de pratiques de complémentation et de constitution de stocks d'ensilage qui visent à sécuriser le système. A l'inverse, d'autres éleveurs recherchent à simplifier la conduite en limitant le rythme de rotation, le nombre de lots ou le chargement. Les logiques sous-tendues peuvent être de réduire les coûts en limitant les intrants ou de simplifier le travail (se dégager du temps pour d'autres activités). Ces pratiques et logiques sous-tendues sont analogues à celles déjà été identifiées en élevage bovin extensif (Josien et al 1994). Ces objectifs intègrent, par ailleurs, les spécifici- tés du contexte européen et ultramarin, en particulier, qui lient fortement le revenu aux aides de l'Etat ou de l'Europe. En prenant ces aides en considération, les éleveurs sont incités à «maximiser» leur nombre de vaches quitte à réduire les performances individuelles. Dans les conditions de la Réunion, cette politique peut permettre d'atteindre des chargements relativement élevés (supérieurs à 2 UGB/ha) sans trop pénaliser la production globale, comme nous l'indique l'étude liée à l'évolution de la PBVV, et d'autant que la réduction des performances avec le chargement peut être compensée par une complémentation des vaches et des broutards comme cela a été démontré expérimentalement (Petit et Muller 1980) et appliquée empiriquement par les éleveurs.

Dans les conditions de la Réunion, où l'agrandissement de l'exploitation n'est pas une voie envisageable, la voie d'amélioration de la productivité est donc celle de la recherche des optima de chargement et de complémentation selon la zone géographique qui soient en adéquation avec les logiques de production identifiées dans notre étude.

\section{Remerciements}

Nous remercions toutes les personnes de l'UAFP (Union des Associations Foncières Pastorales), de l'EDE, de la SicaRévia et du CIRAD ayant participé à l'opération de recherche ainsi que les éleveurs partenaires. Nous remercions également $\mathrm{N}$. Hostiou et $\mathrm{M}$. Naves pour la lecture critique de ce texte.

\section{Références}

Blanfort V., Hassoun P., Mandret G., Paillat J.M. , Tillard E., 2000. L'élevage bovin à la Réunion. Synthèse de quinze ans de recherche. Collection Repères, Cirad/INRA/Région Réunion/CAH, île de la Réunion, France, 350p.

Choisis J.P., Lacroix S., Latchimy J.Y., Legendre E., 2003. La filière viande se structure dans un contexte difficile. Economie de la Réunion, 117, 8-9.

Choisis J.P., Lassalle C., Messad S., Grimaud P., 2008. Performances zootechniques et pratiques des éleveurs en élevage bovin naisseur à la Réunion. Rev. Élev. Méd. Vét. Pays Trop., 61, 89-96.

Diman J.L., Naves M., Marquis K., Alexandre G., Zebus M.F., 2002. Différenciation technicoéconomique des conduites d'élevage bovin viande en Guadeloupe. Renc. Rech. Rum., 9, 126.

Figuié M., 2001. La construction sociale d'un savoir sur la dégradation des ressources naturelles le cas des pâturages dans les exploitations agricoles familiales de la commune de Silvânia au Brésil. Thèse de doctorat de l'INA-PG, 326p.
Grimaud P., Thomas P., 2002. Diversité des rations à base de graminées et gestion des prairies en élevage bovin sur l'île de la Réunion. Fourrages, 169, 65-78.

Grimaud P., Thomas P., 2008. Pasture management and ruminants' meat production in Mascareignes archipelago. World Appl. Sci. J., 3, 763-774.

Grimaud P., Thomas P., Friot D., 2003. Influence of growth of kikuyu (Pennisetum clandestinum) and farmers' practices on dairy and beef production in la Réunion Island. Actes du Symposium régional interdisciplinaire : Les Ruminants, Elevage et Valorisation. Grimaud, P. (Ed.), Région La Réunion/ GDS/Cirad, Saint Denis de la Réunion, 10-13 juin, Synthèse des présentations et CDRom, 42

Hostiou N., Tourrand J.F., Huguenin J., Lecomte P., 2006. La diversité de gestion des systèmes herbagers en Amazonie. Cas des élevages bovins brésiliens. Fourrages, 187, 377392.
Ingrand S., Dedieu B., Agabriel J. 1999. Critères de constitution des lots de vaches des troupeaux de bovins allaitants Limousins et Charolais. INRA Prod. Anim., 12, 61-71.

Institut de l'élevage, 2005. Les systèmes bovins viande en France. Repères techniques et économiques. Synthèse des données recueillies à l'issue de l'exercice 2003 dans les réseaux d'élevage, 20p.

Josien E., Dedieu B., Chassaing C., Babaudou, P. 1994. Réseau extensif bovin limousin : caractéristiques générales des exploitations et éléments de réflexion. Fourrages, 137, 3-23.

Lacroix S., 2002, Etude du fonctionnement des exploitations naisseurs à la Réunion. Mémoire ISTOM, 51p.

Magne M.A., 2007. Modéliser le système d'information des agriculteurs. Le cas des éleveurs de bovins allaitants. Thèse de doctorat, Université Montpellier II, 223p. 
Petit M., Muller A., 1980. Utilisation du pâturage par des vaches allaitantes : influence du chargement. Ann. Zootech., 29, 317-338.
Sarzeaud P., 2002. Coût de production et prix de revient en viande bovine - applications, résultats et limites méthodologiques. Renc. Rech. Rum., 9, 181-184.
Vivier M., 1990. Les prairies et les pratiques d'exploitation. Eléments et réflexions pour un diagnostic. Fourrages, 124, 337-355.

\title{
Résumé
}

Dans les Hauts de la Réunion, l'élevage bovin constitue la principale activité agricole. L'élevage naisseur "professionnel» représente 8828 vaches mères pour 497 exploitations. Ce sont des exploitations de taille modeste (18 vaches mères en moyenne) dont les performances animales sont en-deçà de leurs homologues métropolitaines. L'objectif de l'étude est d'analyser les pratiques des éleveurs naisseurs et d'en faire le lien avec les performances obtenues afin d'identifier des voies d'amélioration potentielles. Pour cela, 10 éleveurs ont fait l'objet d'un suivi de 1999 à 2002, qui était fondé sur l'évaluation des performances des animaux et sur l'enregistrement des pratiques des éleveurs en particularisant l'allotement, la gestion du pâturage et la complémentation alimentaire. Cet article montre que les pratiques sont variées et conditionnées par des objectifs de production différents : stratégies de maximisation des performances ou du chargement, simplification de la conduite.

\begin{abstract}
Livestock farming practices in suckling farm systems in the Highlands of the Reunion island

Cattle farming is the main agricultural activity in the Highlands of the Reunion Island. "Professional" suckling farming represents 8828 cows for 497 farms. They are modest sized farms (18 cows on the average) whose animal performances are lower than their metropolitan counterparts. The aim of the study was to analyse the practices of the suckling farmers with the purpose of improving their management. We carried out herd monitoring on 10 farms from 1999 to 2002 within this framework. This monitoring was based on the measurement of animal performances and on the recording of the farmers' practices in terms of livestock and grazing management. This article presents the analysis carried out on 3 categories of practices: the allotment, grazing management and supplementation. There are various practices underlain by different socioeconomic objectives. Thus, strategies can be founded on the maximisation of the animal performances, the stocking rate or simplification of the management.
\end{abstract}

CHOISIS J.-P., GRIMAUD P., LASSALLE C., 2009. Pratiques d'élevage dans les exploitations bovines allaitantes conduites sur pâturage dans les Hauts de la Réunion. Inra Prod. Anim., 22, 345-354. 